PROCEEDINGS OF THE

AMERICAN MATHEMATICAL SOCIETY

Volume 138, Number 5, May 2010, Pages 1733-1738

S 0002-9939(09)10185-5

Article electronically published on December 16, 2009

\title{
POSITIVE SOLUTIONS OF $q$-DIFFERENCE EQUATION
}

\author{
MOUSTAFA EL-SHAHED AND H. A. HASSAN \\ (Communicated by Varghese Mathai)
}

\begin{abstract}
In this paper we investigate the existence of positive solutions of the $q$-difference equation $-D_{q}^{2} u(t)=a(t) f(u(t))$ with some boundary conditions by applying a fixed point theorem in cones.
\end{abstract}

\section{Preliminaries}

In many of the mathematical models in science such as models of chemical problems, population or concentration in biology, and many problems in physics and economics, we need to investigate the existence of nonnegative solutions. What we understand by nonnegativity can be described by cones, that is, a closed convex set $K$ of a Banach space $X$ such that $\lambda K \subset K$ for all $\lambda \geq 0$ and $K \bigcap(-K)=\{0\}$. Recently there have been many results for positive solutions of different types of boundary value problems. The main task of these results is based on the work of Krasnoselskii [6]. He worked on nonlinear operator equations by using the theory of cones in Banach spaces.

In [2], the authors studied the existence of positive solutions of the second order boundary value problem,

$$
\begin{gathered}
-u^{\prime \prime}=a(t) f(u), \quad 0 \leqslant t \leqslant 1, \\
\alpha u(0)-\beta u^{\prime}(0)=0 \\
\gamma u(1)+\delta u^{\prime}(1)=0,
\end{gathered}
$$

with some conditions imposed on $f(\cdot), a(\cdot)$, and the constants of (2). It is shown that there is a positive solutions in both the superlinear and sublinear cases; see the section below. The authors used a Fixed Point Theorem of Krasnoselskii; see 7 . More precisely, they use a modified version of Krasnoselskii due to Guo [4, p. 94]; it reads:

Theorem 1. Let $\Delta_{1}$ and $\Delta_{2}$ be two bounded open sets in a Banach space $E$ such that $0 \in \Delta_{1}, \overline{\Delta_{1}} \subset \Delta_{2}$. Let

$$
A: K \bigcap\left(\bar{\Delta}_{2} \backslash \Delta_{1}\right) \longrightarrow K
$$

be completely continuous and let one of the conditions

(1) $\|A x\| \leq\|x\|, \quad \forall x \in K \bigcap \partial \Delta_{1}$, and $\|A x\| \geq\|x\|, \quad \forall x \in K \bigcap \partial \Delta_{2} ;$

Received by the editors April 27, 2009, and, in revised form, August 27, 2009.

2010 Mathematics Subject Classification. Primary 39A13, 45M20, 34B18, 34B27.

Key words and phrases. Boundary-value problem, Krasnoselskii's fixed point theorem, Green's function, $q$-difference equation, positive solution.

(C)2009 American Mathematical Society Reverts to public domain 28 years from publication 
(2) $\|A x\| \geq\|x\|, \quad \forall x \in K \bigcap \partial \Delta_{1}$, and $\|A x\| \leq\|x\|, \quad \forall x \in K \bigcap \partial \Delta_{2}$, be satisfied. Then $A$ has at least one fixed point in $K \cap\left(\bar{\Delta}_{2} \backslash \Delta_{1}\right)$.

Here we investigate the analogy of (11)-(2) in the $q$-difference operators case. The constructions and the proofs are closely similar to the case of [2].

In the following section we state main concepts in $q$-calculus and a second order $q$-difference equation with boundary conditions.

\section{INTRODUCTION}

Let $0<q<1$. We say that $A$ is $q$-geometric if for every $x \in A, q x \in A$. Let $f$ be a real or complex valued function defined on a $q$-geometric set $A$. The $q$-difference operator is defined by (cf. [5])

$$
D_{q} f(x):=\frac{f(x)-f(q x)}{x(1-q)}, \quad x \neq 0 .
$$

If $0 \in A$, the $q$-derivative at zero is defined by [1],

$$
D_{q} f(0):=\lim _{n \rightarrow \infty} \frac{f\left(x q^{n}\right)-f(0)}{x q^{n}}, \quad x \in A,
$$

if the limit exists and does not depend on $x$. A right inverse, $q$-integration, of the $q$-difference operator $D_{q}$ is defined by F. H. Jackson (cf. [1, 5]),

$$
\int_{0}^{x} f(t) d_{q} t:=x(1-q) \sum_{n=0}^{\infty} q^{n} f\left(x q^{n}\right), \quad x \in A,
$$

provided that the series converges. In general,

$$
\int_{a}^{b} f(t) d_{q} t:=\int_{0}^{b} f(t) d_{q} t-\int_{0}^{a} f(t) d_{q} t, \quad a, b \in A .
$$

The Leibniz rule and the Fundamental Theorem of $q$-calculus are

$$
\begin{gathered}
D_{q}(f g)(x)=D_{q} f(x) g(x)+f(q x) D_{q} g(x), \\
D_{q}\left(\int_{a}^{x} f(t) d_{q} t\right)=f(x) .
\end{gathered}
$$

Some $q$-functions can be introduced as follows. For $a \in \mathbb{C}$, the symbol $[a]_{q}$ means $[a]_{q}=\left(1-q^{a}\right) /(1-q)$, and the $q$-Pochammer symbol is

$$
(a ; q)_{0}=1, \quad(a ; q)_{k}=\prod_{i=1}^{k-1}\left(1-a q^{i-1}\right), \quad k \in \mathbb{N} \cup\{\infty\},
$$

and

$$
(a ; q)_{\lambda}=\frac{(a ; q)_{\infty}}{\left(a q^{\lambda} ; q\right)_{\infty}}, \quad \lambda \in \mathbb{C} .
$$

The $q$-analogs of sine and cosine functions are defined by

$$
\sin _{q}(x)=\sum_{n=0}^{\infty} \frac{(-1)^{n}}{(q ; q)_{2 n+1}} x^{2 n+1}, \quad \cos _{q}(x)=\sum_{n=0}^{\infty} \frac{(-1)^{n}}{(q ; q)_{2 n}} x^{2 n} .
$$


The reader may find more $q$-functions in 3,8 . These $q$-functions satisfy $q$-difference equations (for example, see [8]),

$$
\begin{aligned}
& \text { if } g(x)=(x ; q)_{\lambda}, \quad \text { then }(1-q)(x-1) D_{q} g(x)-\left(1-q^{\lambda}\right) g(x)=0 ; \\
& \text { if } g(x)=(x ; q)_{\infty}, \quad \text { then }(1-q)(1-x) D_{q} g(x)+g(x)=0 ; \\
& \text { if } g(x)=\sin _{q}(x) \text { or } \cos _{q}(x), \quad \text { then } \quad(1-q)^{2} D_{q}^{2} g(x)+g(x)=0 .
\end{aligned}
$$

The aim of this paper is to establish the existence of positive solutions for the nonlinear second order $q$-difference equation

$$
-D_{q}^{2} u(t)=a(t) f(u(t)), \quad 0 \leqslant t \leqslant 1,
$$

with the boundary conditions

$$
\begin{aligned}
& \alpha u(0)-\beta D_{q} u(0)=0, \\
& \gamma u(1)+\delta D_{q} u(1)=0,
\end{aligned}
$$

where $\rho:=\gamma \beta+\alpha \gamma+\alpha \delta>0, \alpha, \beta, \gamma, \delta \geq 0$. The functions $a(\cdot), f(\cdot)$ are assumed to be nonnegative continuous functions for $t \in[0,1]$, and $a(t) \not \equiv 0$ on any subinterval of $[0,1]$. We also assume that $f(\cdot)$ is either superlinear or sublinear. These definitions can be defined as follows. Let

$$
f_{0}:=\lim _{u \rightarrow 0} \frac{f(u)}{u}, \quad f_{\infty}:=\lim _{u \rightarrow \infty} \frac{f(u)}{u} .
$$

Then the superlinear case means $f_{0}=0, f_{\infty}=\infty$, and the other one means $f_{0}=\infty, f_{\infty}=0$.

\section{Green's Function And existence theorem}

In this section we construct the solution of (8)-(9) in a form of $q$-integral transform involving Green's Function. This Green's Function takes the same form of the classical case if $q=1$. Then we give some estimations and inequalities for this Green's Function which will be the main tools in getting the positive solution of (8) - (9)

Lemma 1. Any solution of (8) -(9) is

$$
u(t)=\int_{0}^{1} G(t, q s) a(s) f(u(s)) d_{q} s,
$$

where $G(t, s)$ is the Green's function of $-D_{q}^{2} u(t)=0$ with (9), which is

$$
G(t, s)=\frac{1}{\rho} \begin{cases}(\gamma+\delta-\gamma t)(\beta+\alpha s), & 0 \leq s \leq t \leq 1 \\ (\gamma+\delta-\gamma s)(\beta+\alpha t), & 0 \leq t \leq s \leq 1\end{cases}
$$

Proof. Using the variation of parameters method, the solution of (8) has the form

$$
u(t)=c_{1} t+c_{2}+\int_{0}^{t}[t-q s] a(s) f(u(s)) d_{q} s,
$$

where $c_{1}, c_{2}$ are arbitrary constants. For $u$ satisfying (9), using (6)-(7) we get

$$
\begin{aligned}
& c_{1}=-\frac{\alpha}{\rho} \int_{0}^{1}[(\gamma+\delta)-\gamma q s] a(s) f(u(s)) d_{q} s, \\
& c_{2}=-\frac{\beta}{\rho} \int_{0}^{1}[(\gamma+\delta)-\gamma q s] a(s) f(u(s)) d_{q} s .
\end{aligned}
$$


Substituting in (12) we obtain the form (10).

Clearly $G(t, q s) \geq 0$. For our purpose we also need the following estimation of $G(t, q s)$.

Lemma 2. Let

$$
\sigma=\min \left\{\frac{\gamma+4 \delta}{4(\gamma+\delta)}, \frac{\alpha+4 \beta}{4(\alpha q+\beta)}\right\}
$$

Then

$$
G(t, q s) \geq \sigma G(q s, q s), \quad \frac{1}{4} \leq t \leq \frac{3}{4}, \quad 0 \leq s \leq 1 .
$$

Also

$$
G(t, q s) \leq G(q s, q s), \quad 0 \leq t, s \leq 1 .
$$

Proof. If $1 / 4 \leq t \leq 3 / 4$, then

$$
\frac{G(t, q s)}{G(q s, q s)}= \begin{cases}\frac{\gamma+\delta-\gamma t}{\gamma+\delta-\gamma q s} \geq \frac{\gamma+4 \delta}{4(\gamma+\delta)}, & q s \leq t, \\ \frac{\beta+\alpha t}{\beta+\alpha q s} \geq \frac{\alpha+4 \beta}{4(\alpha q+\beta)}, & q s \geq t .\end{cases}
$$

Thus (13) follows. Also (14) follows from

$$
\frac{G(t, q s)}{G(q s, q s)}=\left\{\begin{array}{l}
\frac{\gamma+\delta-\gamma t}{\gamma+\delta-\gamma q s} \leq 1, \quad q s \leq t, \\
\frac{\beta+\alpha t}{\beta+\alpha q s} \leq 1, \quad q s \geq t .
\end{array}\right.
$$

Now let $A: X \longrightarrow X, X=C[0,1]$, be defined by

$$
A u(t)=\int_{0}^{1} G(t, q s) a(s) f(u(s)) d_{q} s .
$$

Define the cone $K$ in $X$ by

$$
K=\left\{u \in X: u(t) \geq 0, \min _{1 / 4 \leq t \leq 3 / 4} u(t) \geq \sigma\|u\|\right\} .
$$

Lemma 3. $A$ is a positive operator, that is,

$$
A(K) \subset K \text {. }
$$

Proof. Clearly $A(u) \geq 0$ for $u \in K$. Using Lemma 2, we get

$$
\begin{aligned}
\min _{1 / 4 \leq t \leq 3 / 4} A u(t) & =\min _{1 / 4 \leq t \leq 3 / 4} \int_{0}^{1} G(t, q s) a(s) f(u(s)) d_{q} s \\
& \geq \sigma \int_{0}^{1} G(q s, q s) a(s) f(u(s)) d_{q} s \\
& \geq \sigma \max _{0 \leq t \leq 1} \int_{0}^{1} G(t, q s) a(s) f(u(s)) d_{q} s \\
& =\sigma\|A u\| .
\end{aligned}
$$

Theorem 2. The problem (8) - (9) has at least one positive solution in the superlinear and sublinear cases. 
Proof. First we consider the superlinear case. Since $f_{0}=0$, for $\epsilon$ satisfying

$$
0<\epsilon \int_{0}^{1} G(q s, q s) a(s) d_{q} s \leq 1,
$$

we can choose $\delta_{1}>0$ such that $f(u) \leq \epsilon u$, for $0<u \leq \delta_{1}$. Thus, if also $u \in K$, then from Lemma 2 we have

$$
A u(t)=\int_{0}^{1} G(t, q s) a(s) f(u(s)) d_{q} s \leq \int_{0}^{1} G(q s, q s) a(s) \epsilon u(s) d_{q} s \leq\|u\| .
$$

Define the set

$$
\Delta_{1}:=\left\{u \in X:\|u\|<\delta_{1}\right\} .
$$

Thus from (20), we get

$$
\|A u\| \leq\|u\|, \quad u \in K \cap \partial \Delta_{1} .
$$

Also, because of $f_{\infty}=\infty$, there exists $\delta^{*}$ such that $f(u) \geq \mu u, u \geq \delta^{*}$, where $\mu$ satisfies

$$
\mu \sigma \int_{1 / 4}^{3 / 4} G(1 / 2, q s) a(s) d s \geq 1 .
$$

Let $\delta_{2}=\max \left\{2 \delta_{1}, \delta^{*} / \sigma\right\}$, and let

$$
\Delta_{2}:=\left\{u \in X:\|u\|<\delta_{2}\right\} .
$$

Therefore for $u \in K$ and $\|u\|=\delta_{2}$, we have

$$
\min _{1 / 4 \leq t \leq 3 / 4} u(t) \geq \sigma\|u\| \geq \delta^{*} .
$$

Thus

$$
\begin{aligned}
A u(1 / 2) & =\int_{0}^{1} G(1 / 2, q s) a(s) f(u(s)) d s=\int_{1 / 4}^{3 / 4} G(1 / 2, q s) a(s) f(u(s)) d s \\
& \geq \mu \int_{1 / 4}^{3 / 4} G(1 / 2, q s) a(s) u(s) d s \geq \mu \sigma\|u\| \int_{1 / 4}^{3 / 4} G(1 / 2, q s) a(s) d s \geq\|u\| .
\end{aligned}
$$

This means $\|A u\| \geq\|u\|$ for $u \in K \cap \partial \Delta_{2}$.

Hence, by the fixed point theorem, there exists a fixed point $u$ of $A$ where $u \in K \cap\left(\bar{\Delta}_{2} \backslash \Delta_{1}\right), \delta_{1} \leq\|u\| \leq \delta_{2}$.

For the sublinear case $\left(f_{0}=\infty, f_{\infty}=0\right)$ we can choose $\delta_{1}^{\prime}>0$ such that $f(u) \geq \mu^{\prime} u$, for $0<u \leq \delta_{1}$, where

$$
\mu^{\prime} \sigma \int_{1 / 4}^{3 / 4} G(1 / 2, q s) a(s) d s \geq 1 .
$$

Hence, if $u \in K$ and $\|u\|=\delta_{1}^{\prime}$, we get in a way similar to the first case $A u(1 / 2) \geq$ $\|u\|$ and

$$
\|A u\| \geq\|u\|, \quad u \in K \cap \partial \Delta_{1}^{\prime}, \Delta_{1}^{\prime}:=\left\{u \in X:\|u\|<\delta_{1}^{\prime}\right\} .
$$

Since $f_{\infty}=0$, there is a $\widetilde{\delta}$ such that $f(u) \leq \epsilon^{\prime} u$, for $u \geq \widetilde{\delta}$, where

$$
0<\epsilon^{\prime} \int_{0}^{1} G(q s, q s) a(s) d_{q} s \leq 1 .
$$

Let $\delta_{2}^{\prime}=\max \left\{2 \delta_{1}^{\prime}, \widetilde{\delta} / \sigma\right\}$ and

$$
\Delta_{2}^{\prime}:=\left\{u \in X:\|u\|<\delta_{2}^{\prime}\right\}
$$


Then for $u \in K$ and $\|u\|=\delta_{2}^{\prime}$, we have

$$
\min _{1 / 4 \leq t \leq 3 / 4} u(t) \geq \sigma\|u\| \geq \widetilde{\delta}
$$

Hence, for $u \in K$ and $\|u\|=\delta_{2}^{\prime}$, we get

$$
\|A u\| \leq\|u\|, \quad u \in K \cap \partial \Delta_{2}^{\prime} .
$$

Therefore, $A$ has a fixed point.

\section{REFERENCES}

[1] M. H. Annaby and Z. S. Mansour, $q$-Taylor and interpolation series for Jackson $q$-difference operators, Journal of Mathematical Analysis and Applications, 344: 472-483, 2008. MR.2416321 (2009b:33020)

[2] L.H. Erbe and H. Wang, On the existence of positive solutions of ordinary differential equations. Proc. Amer. Math. Soc., 120: 743-748, 1994. MR.1204373 (94e:34025)

[3] G. Gasper and M. Rahmen, Basic Hypergeometric Series, Cambridge University Press, Cambridge, 2004. MR2128719 (2006d:33028)

[4] D. Guo and V. Lakshmikantham, Nonlinear Problems in Abstract Cones, Academic Press, San Diego, 1988. MR959889 (89k:47084)

[5] F.H. Jackson, On q-definite integrals. Quart. J. Pure and Appl. Math., 41: 193-203, 1910.

[6] M. A. Krasnoselskii, Positive Solutions of Operator Equations, Noordhoff, Groningen, 1964. MR0181881(31:6107)

[7] M. K. Kwong, On Krasnoselskii's cone fixed point theorem. Fixed Point Theory and Applications, 2008, Art. ID 164537, 1-18. MR2395322 (2009d:47066)

[8] S. D. Marinković, P. M. Rajković and M. Stanković, The Linear $q$-Differential Equation and $q$-Holonomic Functions, XVII Conference on Applied Mathematics, D. Herceg, H. Zarin, eds.; Department of Mathematics and Informatics, Novi Sad, 2007, 13-20.

College of Education, P.O. Box 3771, Qasssim - Unizah, Kingdom of Saudi Arabia

E-mail address: elshahedm@yahoo.com

Department of Mathematics, Faculty of Basic Education, The Public Authority for Applied Education and Training, P.O. Box 23167, Kuwait

E-mail address: hassanatef1@gmail.com 